\title{
Split gate and asymmetric contact carbon nanotube optical devices
}

\author{
M. A. Hughes, ${ }^{* a}$ K. P. Homewood ${ }^{\mathrm{a}}$, R. J. Curry ${ }^{\mathrm{a}}$, Y. Ohno ${ }^{\mathrm{b}}$ and T. Mizutani ${ }^{\mathrm{b}}$ \\ ${ }^{a}$ Advanced Technology Institute, Department of Electronic Engineering, University of Surrey, \\ Guildford, GU2 7XH, United Kingdom; ' Department of Quantum Engineering, Nagoya University, \\ Furo-cho, Nagoya, 464-8603, Japan
}

\begin{abstract}
.
Asymmetric contacts or split gate geometries can be used to obtain rectification, electroluminescence (EL) and photocurrent from carbon nanotube field effect transistors. Here, we report devices with both split gates and asymmetric contacts and show that device parameters can be optimised with an appropriate split gate bias, giving the ability to select the rectification direction, modify the reverse bias saturation current and the ideality factor. When operated as a photodiode, the short circuit current and open circuit voltage can be modified by the split gate bias, and the estimated power conversion efficiency was $1 \times 10^{-6}$. When using split gates and symmetric contacts, strong EL peaking at $0.86 \mathrm{eV}$ was observed with a full width at half maximum varying between 64 and $120 \mathrm{meV}$, depending on the bias configuration. The power and quantum efficiency of the EL was estimated to be around $1 \times 10^{-6}$ and $1 \times 10^{-5}$ respectively.
\end{abstract}

Keywords: carbon nanotube, split gate, asymmetric contact, electroluminescence, photocurrent

*Electronic mail: m.a.hughes@surrey.ac.uk

\footnotetext{
Optical Components and Materials XI, edited by Michel J. F. Digonnet, Shibin Jiang,

Proc. of SPIE Vol. 8982, 89820P · (c) 2014 SPIE · CCC code: 0277-786X/14/\$18

doi: $10.1117 / 12.2036962$

Proc. of SPIE Vol. $898289820 \mathrm{P}-1$
} 


\section{Introduction}

Carbon nanotubes (CNTs) are one-dimensional materials with exceptional electronic properties. Their charge carrier mobility can be as high as $100000 \mathrm{~cm}^{2} / \mathrm{Vs}$ [1], and their current carrying capacity can exceed $1 \times 10^{9} \mathrm{~A} / \mathrm{cm}^{2}$ [2]. Electroluminescence (EL) from CNTs may have applications in nanoscale lasers [3], single photon sources [4], quantum computing [5], and optical communications [6]. In ambipolar devices, the light emitting recombination region can be moved along the CNT using the gate voltage [7]. This effect could be exploited in optical communication systems for multiplexing a signal over multiple channels. Electrostatic doping is a novel technique that through the use of a split gate, has been used to demonstrate CNT field effect transistor (FET) p-n junction diodes [3, 8-11]. It can also be used to modify the band profile of CNTs to facilitate EL. CNTs also offer potential advantages in photovoltaic applications, since the bandgap of a CNT is directly related to its chiral angle and diameter [12] and can be chosen to match a wide range of the solar spectrum [13]. Recently, efficiencies of $>11 \%$ have been demonstrated in p-type CNT/ n-type Si hetrojunction solar cells [14] and 14\% in nitric acid wetted CNT/Si hetrojunction solar cells [15]. CNT based photodetectors may also have the ability to support multiple exciton generation which could drastically improve power conversion efficiencies [16]. Photocurrent imaging of CNT photodiodes shows that the detecting region can be tuned with the gate bias [17]. Photodiodes typically have a trade-off between detection bandwidth and efficiency; this trade-off may be overcome by the ability to control the built-in voltage $\left(\mathrm{V}_{\mathrm{bi}}\right)$ of the photodiode.

In this work we report that the electrical and optical properties CNT FETs with split gate geometry can be enhanced and tuned by applying specific split gate biases. With asymmetric contacts strong rectification is observed and both the rectification direction and saturation current can be modified using the split gate bias. These devices display one of the lowest reported reverse bias saturation currents for a CNT diode, as well as one of the highest reported charge carrier mobility and on/off ratio combinations of a CNT FET. When the device is operated as a photodiode, we report tuning of the open circuit voltage $\left(\mathrm{V}_{\mathrm{oc}}\right)$, and therefore $\mathrm{V}_{\mathrm{bi}}$, and the short circuit current $\left(\mathrm{I}_{\mathrm{sc}}\right)$ with a split gate bias. Two types of device were fabricated: (i) a symmetric device in which both source and drain contacts were Ti; and (ii) an asymmetric device in which the source contact was Ti and the drain contact was Pd. A number of device geometries were fabricated by varying the gate to gate distance (G-G) between 1 to $3 \mu \mathrm{m}$ and the source/drain to gate distance (S-G) between 0.3 to $1 \mu \mathrm{m}$, see figure 1a. Channel widths of 10,50 or $100 \mu \mathrm{m}$ were also fabricated in order to control the number of CNTs 
bridging the channel. In this paper we focus on two devices, the first being a symmetric device with a G-G distance of 1 $\mu \mathrm{m}$, a G-S distance of $0.5 \mu \mathrm{m}$ and a channel width of $100 \mu \mathrm{m}$. The second device is an asymmetric device with a G-G distance of $1 \mu \mathrm{m}$, a G-S distance of $0.3 \mu \mathrm{m}$ and a channel width of $10 \mu \mathrm{m}$. The SEM images in figure $1 \mathrm{~b}$ and $\mathrm{c}$ show these two devices. Seven CNTs were observed to bridge the channel in the case of the symmetric device and only one CNT in the asymmetric case. The dependence of $I_{d} V_{d s}$ characteristics, EL and photocurrent on G-G and G-S distance was found to be weak but depended strongly on the number of nanotubes bridging the channel. EL could only be observed when multiple nanotubes bridged the channel whilst photocurrent could only be observed in single nanotube devices when the reverse bias current was low.

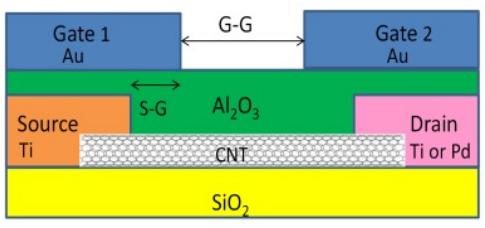

(a)

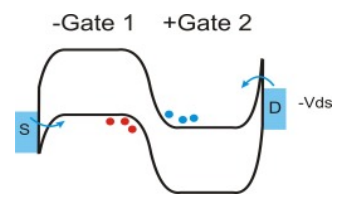

(d)

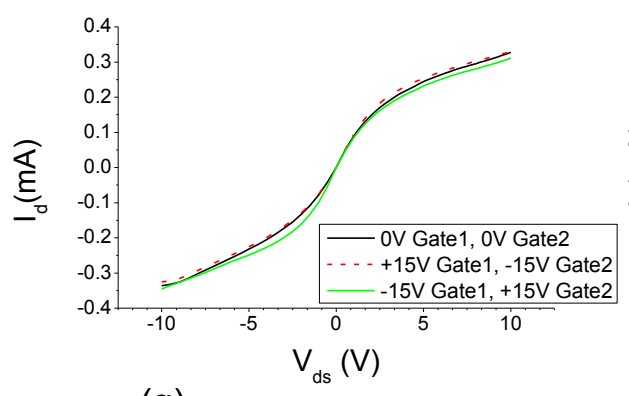

(g)

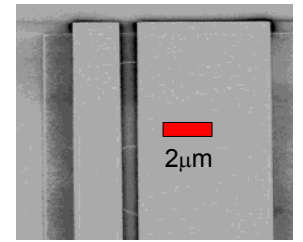

(b)

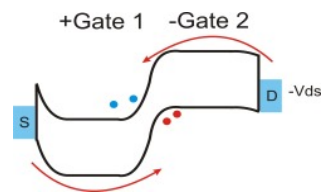

(e)

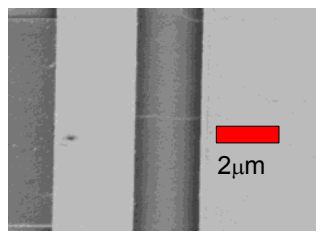

(c)

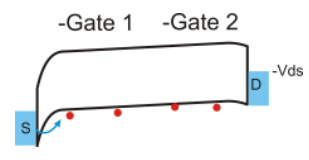

(f)

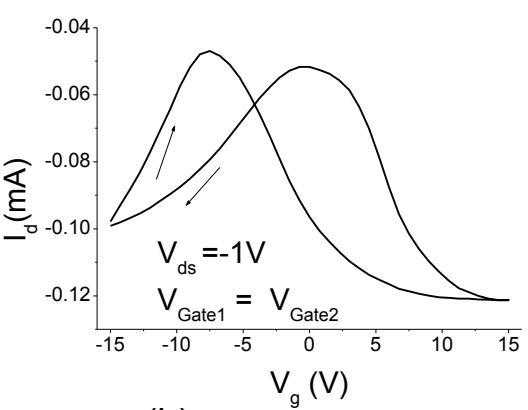

(h)

Figure 1. Schematic cross-section of the device structures, the symmetric device had Ti-Ti contacts, whereas the asymmetric device had Ti-Pd contacts (a). SEM image of the symmetric device, seven CNTs were observed bridging the channel (b). SEM image of the asymmetric device, one CNT was observed bridging the channel (c). Band diagram representing the symmetric device in forward bias 
configuration with gate $1,2=-15,+15 \mathrm{~V}$, the blue arrows illustrate the greater significance of tunnelling for electron transport in this configuration (d). Band diagram representing the symmetric device in reverse bias configuration with gate $1,2=+15,-15 \mathrm{~V}$, the red arrows illustrate the greater significance of thermionic emission for electron transport in this configuration(e). Band diagram representing the symmetric device with equal split gate bias, which illustrates how carriers can flow freely in this configuration (f), rather than becoming trapped by band bending, as in (d) and (e). $I_{d^{-}}-V_{d s}$ characteristics of the symmetric device at various split gate biases (g). Transfer characteristic with equal split gate bias and $\mathrm{V}_{\mathrm{ds}}=-1 \mathrm{~V}(\mathrm{~h})$.

\section{Experimental}

Devices were fabricated by growing SWNTs from $0.1 \mathrm{~nm}$ of Co catalyst, patterned by photolithography, on a $\mathrm{SiO}_{2} / \mathrm{Si}$ wafer by ethanol chemical vapour deposition (CVD) at $800{ }^{\circ} \mathrm{C}$. The catalyst was first activated in air at $800{ }^{\circ} \mathrm{C}$. This method produces well separated SWNTs with diameters of 0.5 to $2 \mathrm{~nm}$ [18-21]. Electron beam (EB) lithography was used to pattern source and drain contacts, which were deposited by EB evaporation of $30 \mathrm{~nm}$ of Pd or Ti. A gate insulator was formed by atomic layer deposition (ALD) of $40 \mathrm{~nm}$ of $\mathrm{Al}_{2} \mathrm{O}_{3}$ at $300{ }^{\circ} \mathrm{C}$. The split gates (gates 1 and 2) were fabricated by EB lithography and deposition of Ti/Au. $I_{d^{-}} V_{d s}$ scans were obtained using an Agilent B1500 SCS with a minimum current detection limit in our test setup of around $100 \mathrm{fA}$. SEM images were taken using a Zeiss Evo50 at $2 \mathrm{kV}$ using in-line lenses. As the CNTs in our devices are encapsulated beneath $40 \mathrm{~nm}$ of $\mathrm{Al}_{2} \mathrm{O}_{3}$, it is unlikely that the SEM images are derived purely from secondary electrons; we propose that the contrast mechanism observed in the image results from charge build up in the nanotube and its metal contacts, leading to enhanced secondary electron yield directly above the CNT. A similar contrast mechanism is thought to occur with bare CNTs on insulating substrates [22]. EL measurements were taken in air by collecting the EL with a 50x objective with a focal length of $\sim 1 \mathrm{~cm}$, the EL was then dispersed by a monochromator and detected using a liquid nitrogen cooled InGaS detector array. Photocurrent measurements were obtained using a focused a Ti:sapphire laser tuned to $735 \mathrm{~nm}$ with a measured spot diameter of $4 \mu \mathrm{m}$ on to the device. The laser power was varied using a neutral density filter.

\section{Results and discussion}

\subsection{Symmetric contact device}

The electrostatic doping technique can form a rectifying $\mathrm{p}-\mathrm{n}$ junction by doping adjacent sections of a CNT $\mathrm{p}$ or $\mathrm{n}$ type through application of opposite bias to the split gates $[3,8]$. In this situation the device is in forward bias when gate 2 has 
the opposite polarity to $V_{d s}$, as illustrated in Figure 1d. The device is in reverse bias when gate 2 has the same polarity as $V_{d s}$, as illustrated in Figure 1e. Figure $1 \mathrm{~g}$ shows the $I_{d^{-}} V_{d s}$ characteristics of the symmetric contact (Ti:Ti) device. The $I_{d^{-}}$ $V_{d s}$ characteristics are largely symmetric and in both forward and reverse bias configuration there is a respective increase or decrease in $I_{d}$ of $\sim 5 \%$. The relatively high current in reverse bias could indicate that a large proportion of carriers are transported across the Schottky barrier by thermionic emission. In forward bias we would expect a greater proportion of carriers to be transported by tunnelling as is illustrated by the arrows in figure $1 \mathrm{~d}$ and e. Figure $1 \mathrm{~h}$ shows the transfer characteristics with equal gate bias. The device shows ambipolar characteristics, indicating the ability to inject holes and electrons. The on-off ratio is $\sim 2$ and we suggest that the off-state current is caused either by the presence of metallic CNTs, or the superposition of transfer characteristics of multiple CNTs with different off-state gate potentials. Removal of possible metallic CNTs by electrical breakdown was not possible due to encapsulation of the CNTs, which meant there was a current large enough to destroy the device before any breakdown of CNTs. The hysteresis in the transfer characteristics indicates the presence of trapped charges (holes or electrons) in the substrate and/or gate insulator [23, 24]. Comparing the increase in $I_{d}$ with the application of a gate bias, Figure $1 \mathrm{~g}$ and $\mathrm{h}$, shows that modulation depth of the gate is significantly larger when the gates have equal rather than opposite polarity. In order to explain this we propose that when opposite gate polarities are applied to the device carriers can become trapped by band bending, as illustrated in Figure $1 \mathrm{~d}$ and e, resulting in the $\sim 5 \%$ increase of $\mathrm{I}_{\mathrm{d}}$ when using opposite gate bias observed in Figure $1 \mathrm{~g}$. Whereas when equal gate bias of the same polarity is applied to the device carriers can move freely, as illustrated in Figure 1f, resulting in the $\sim 100 \%$ increase of $I_{d}$ when using equal gate bias observed in Figure 1h. A similar effect has been illustrated, but not explained, in comparable device structures [25].

Proc. of SPIE Vol. $898289820 \mathrm{P}-5$ 


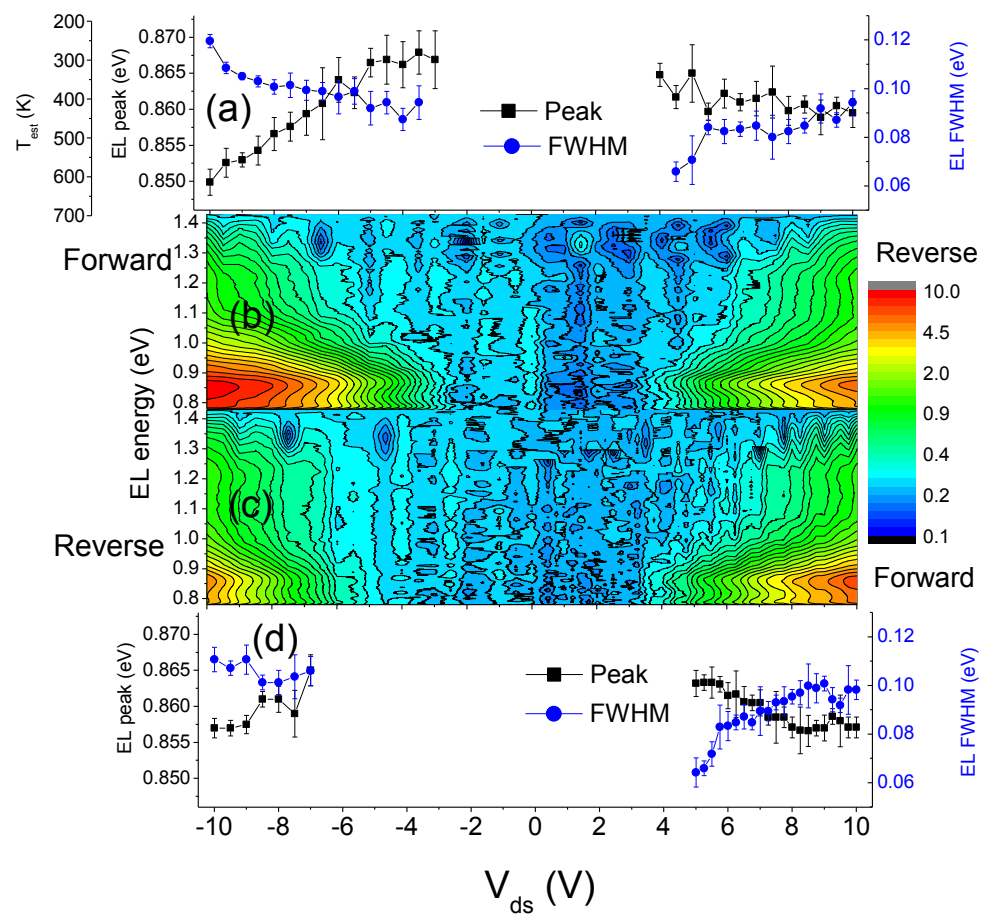

Figure 2. Contour plot of EL spectra as a function of drain bias for gate $1,2=-15 \mathrm{~V},+15 \mathrm{~V}$ (b) and gate $1,2=+15 \mathrm{~V},-15 \mathrm{~V}$, (c) each forward or reverse bias configuration is marked, the intensity is in a log scale. The peak position and FWHM of the main EL peak, derived from deconvolution of the EL spectra into Gaussians, are shown for gate $1,2=-15 \mathrm{~V},+15 \mathrm{~V}$ (a) and gate $1,2=+15 \mathrm{~V},-15 \mathrm{~V}$ (d). The lattice temperature $\left(T_{\text {est }}\right)$, estimated from the shift of the EL peak position, is shown on a second y-axis in (a).

Figure 2 shows contour plots of EL spectra as a function of drain bias for gate 1,2 = -15, $+15 \mathrm{~V}$ (b) and gate $1,2=+15 \mathrm{~V}$, $-15 \mathrm{~V}$ (c), constituting four forward or reverse configurations. There is a strong EL peak at $\sim 0.86 \mathrm{eV}$; for a CNT emitting at this energy, embedded in $\mathrm{Al}_{2} \mathrm{O}_{3} / \mathrm{SiO}_{2}\left(\varepsilon_{\mathrm{eff}} \approx 5.7\right)$, we estimate an exciton binding energy of $0.12 \mathrm{eV}$ [26], giving an estimated CNT diameter of $\sim 1.05 \mathrm{~nm}[12,27]$. There are also several other weaker EL bands extending up to $1.2 \mathrm{eV}$. The weakness of the higher energy bands could be due to the difficulty of electrically exciting wide bandgap single walled nanotubes (SWNTs) [28]. The results in Figure 3 indicate that EL produced with a split gate bias is generated by an ambipolar mechanism, i.e. from the recombination of holes and electrons. The EL intensity can therefore be seen as a proxy for the recombination current. The strength of the EL for the various forward and reverse bias configurations can be represented as three levels: forward bias in Figure $2 \mathrm{~b}$ is strong, reverse bias in Figure $2 \mathrm{c}$ is weak, and reverse bias in Figure $2 \mathrm{~b}$ and forward bias in Figure $2 \mathrm{c}$ are both intermediate. This asymmetry of the EL intensity for the different 
forward and reverse bias configurations can be explained by the high drain bias, which implies that the total deflection of the conduction and valence bands by the gate is proportional to the sum of the gate and drain biases at the drain, but only the gate potential at the source. This is illustrated in the band diagram in Figure 1d. This results in electron injection into the CNT by tunnelling being easiest for the forward bias configuration in Figure 2b, most difficult for the reverse bias configuration in Figure 2c, and intermediate for configurations of reverse bias in Figure $2 b$ and forward bias in Figure $2 c$.

Figure 2a and d show the EL FWHM as a function of $V_{d s}$. In figure 2a it can be seen that the minimum EL FWHM is lower in reverse bias $(66 \mathrm{meV})$ than in forward bias $(87 \mathrm{meV})$. The forward bias region of figure $2 \mathrm{a}$ and the reverse bias region of figure $2 \mathrm{~d}$ show an accelerating and decelerating dependence on the magnitude of $V_{d s}$ respectively. The EL FWHM is lowest for positive $V_{d s}$ with both gate configurations, indicating that hole injection promotes a smaller FWHM. We expect changes in the EL peak position and FWHM with increasing $V_{d s}$ to be related to Joule heating which should cause a red shift in the EL peak position [29] and broadening of the EL peak due to increased thermal and lifetime broadening effects [30]. The bandgap $\left(\mathrm{E}_{\mathrm{g}}\right)$ for most SWNTs decreases linearly with temperature with a slope of around $5 \times 10^{-5} \mathrm{eV} /{ }^{\circ} \mathrm{C}[31]$, enabling the shift in EL peak position to be used to estimate lattice temperature $\left(\mathrm{T}_{\text {est }}\right)$. As $V_{d s}$ increases from 3 to $5 \mathrm{~V}$ in the forward bias region of Figure 2a, the main EL peak and FWHM remain relatively unchanged, indicating that no significant heating of the CNT occurs in this voltage range. For $V_{d s}$ biases above $5 \mathrm{~V}$ the main EL peak shifts to lower energy and broadens. We assumed the lattice temperature is around $300 \mathrm{~K}$ in the 3 to $5 \mathrm{~V} V_{d s}$ range where the EL peak is $\sim 0.867 \mathrm{eV}$. $\mathrm{T}_{\text {est }}$ was then calculated from the peak shift $(\Delta \mathrm{EL})$ by $\mathrm{T}_{\text {est }}=\left(\Delta \mathrm{EL} / 5 \times 10^{-5}\right)+300$. As shown in Figure 2a, the lattice temperature reached an estimated $650 \mathrm{~K}$ at $\mathrm{V}_{\mathrm{ds}}=-10 \mathrm{~V}$.

At $V_{d s}=-10 \mathrm{~V}$, and gate $1,2=-15 \mathrm{~V},+15 \mathrm{~V}$, an excellent fit of the EL spectrum was obtained using a sum of 5 Gaussian emitters, see figure A.1. We also identified a low energy band that lies mostly outside the detector range, but can be seen as a deviation from the Gaussian fit. This indicates a total of six EL emitters. We suggest that each band originates from an individual CNT which is in good agreement with the SEM observation of seven CNTs bridging the channel. The unaccounted CNT may be metallic or poorly contacted. By comparing the EL intensity of the device to that of a chip LED, with a known efficiency, we estimate the maximum power and quantum efficiency of the EL to be around $1 \times 10^{-6}$ and $1 \times 10^{-5}$, respectively. This compares to quantum efficiencies of $1 \times 10^{-4}$ in single CNT split gate diodes [3], $1 \times 10^{-10}$ to $1 \times 10^{-9}$ for electrolyte gated CNT FETs [6], and $1 \times 10^{-6}$ for CNT network transistors [32]. The FWHM of the main EL 
peak varied between 64 and $120 \mathrm{meV}$ over all bias configurations. Typically, an EL FWHM > $150 \mathrm{meV}$ indicates hot carriers are involved in EL process [33], hence the EL FWHM observed above suggests that hot carriers are not involved.

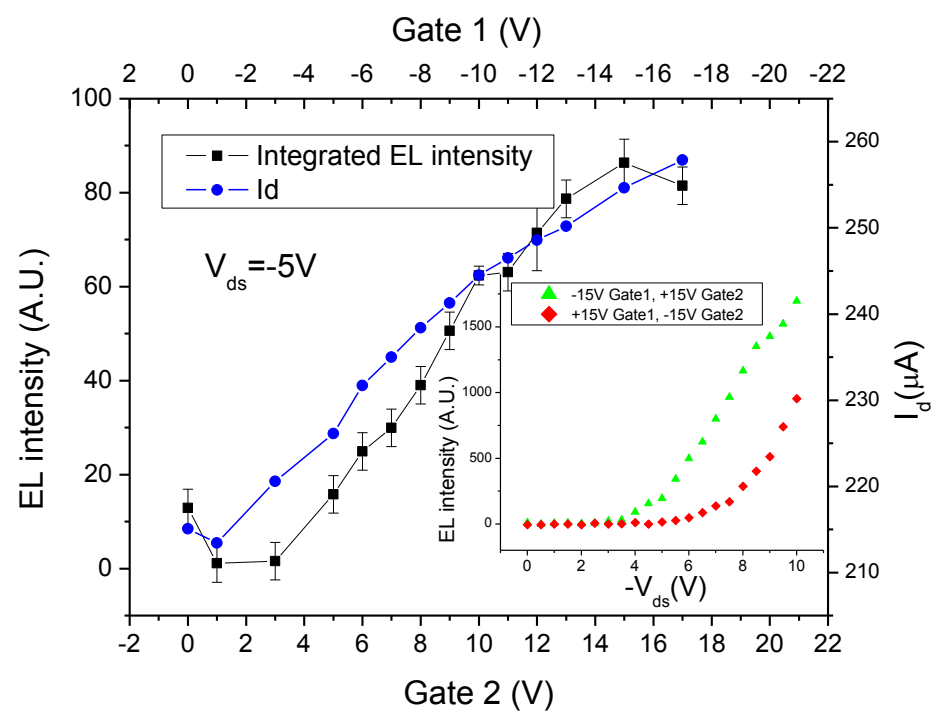

Figure 3. Integrated EL intensity and drain current as a function of split gate bias, with a drain bias fixed at $-5 \mathrm{~V}$. Inset shows the integrated EL intensity as a function of drain bias with split gate biases of gate1,2 $=-15,+15 \mathrm{~V}$ (corresponding to forward bias) and gate $1,2=+15 \mathrm{~V},-15 \mathrm{~V}$ (corresponding to reverse bias). When integrated EL intensity is plotted as a function of $I_{d}$, the dependence is broadly similar.

Figure 3 shows the integrated EL intensity as a function of increasing opposite polarity split gate bias, with $V_{d s}=-5 \mathrm{~V}$, putting the device into an increasing forward bias configuration. There is a small amount of EL at zero gate bias which should be due to a unipolar mechanism. The EL intensity then reduces to zero with increasing opposite polarity split gate bias before increasing approximately linearly with split gate bias. Unipolar EL can occur near trapped charges in the substrate or gate insulator, defects, CNT contacts, or any other inhomogeneities that produce a local electric field capable of accelerating carriers above a threshold energy at which electronic excitation of the CNT across the bandgap can take place [34]. Based on explanations of a similar effect [35], we propose that a pocket of electrons trapped in the insulator dopes a small segment of the CNT p-type. Under zero gate bias we can expect n-type conduction, see Figure 1h, which would then produce an n-p-n junction which produces the unipolar EL. When gate 1 dopes this section of the CNT ptype the unipolar EL disappears, then, with increasing split gate bias opposite ends of the CNT are doped n and $\mathrm{p}$ type respectively. These carriers recombine to produce much stronger EL by an ambipolar mechanism. In Figure $3, I_{d}$ 
increases by a relatively small amount from $215 \mu \mathrm{A}$ to $255 \mu \mathrm{A}$ when the split gate bias is increased from zero to $+/-17 \mathrm{~V}$. At zero gate bias we suppose that $I_{d}$ consists mostly of majority carriers, and the increase in $I_{d}$ caused by the split gate bias is due to the injection of minority carriers, which can produce EL by an ambipolar mechanism. The inset of Figure 3 shows the integrated electroluminescence intensity as a function of $V_{d s}$ for split gate biases of gate $1,2=-15,+15 \mathrm{~V}$ (forward bias) and gate 1,2 $=+15,-15 \mathrm{~V}$ (reverse bias). It shows that EL is stronger in forward bias than reverse bias for the same $V_{d s}$. Forward bias should promote e-h pair recombination and therefore the increase in EL may be due to e-h pair recombination. EL in reverse bias should originate from a unipolar mechanism. The general trend of EL intensity is similar when it is plotted as a function of input power $\left(I_{d} V_{d s}\right)$ or $I_{d}$, with each plot showing a linear relationship above a threshold. A linear dependence of EL intensity on $\mathrm{I}_{\mathrm{d}}$ has also been reported for ambipolar CNT FETs [28]. It has been reported that a thresholdless dependence of EL power on electrical power indicates e-h pair recombination by an ambipolar mechanism [25]. The presence of a threshold for EL indicates a unipolar mechanism occurs, where electrons or holes are accelerated to energies sufficient to generate excitons by impact excitation [25, 34]. However, the gate dependence of EL in Figure 3 indicates the presence of a p-n junction and an ambipolar mechanism. We propose that this apparent contradiction occurs because there is a larger Schottky barrier height for holes than for electrons This would result in the EL intensity being dependent on the hole current, which we would expect to have a threshold-like dependence on $V_{d s}$.

\subsection{Asymmetric contact device}

Figure 4 shows the $I_{d}-V_{d s}$ characteristics of the asymmetric device at various applied split gate biases. In contrast to the symmetric devices, which had broadly symmetric $I_{d}-V_{d s}$ characteristics regardless of the gate bias, the asymmetric contact device displays strong rectification. This can be explained by the different work function of metal contacts which could suppress carrier injection in reverse bias. The Fermi level of the CNT in our device should be close to the middle of the bandgap, as indicated by the transfer characteristics in figure 5. To extract diode parameters, $I_{0}$ (saturation current), $\eta$ (ideality factor) and $R_{\mathrm{s}}$ (series resistance), $I_{d^{-}} V_{d s}$ curves were fitted using the method detailed ref [36].

Proc. of SPIE Vol. $898289820 \mathrm{P}-9$ 


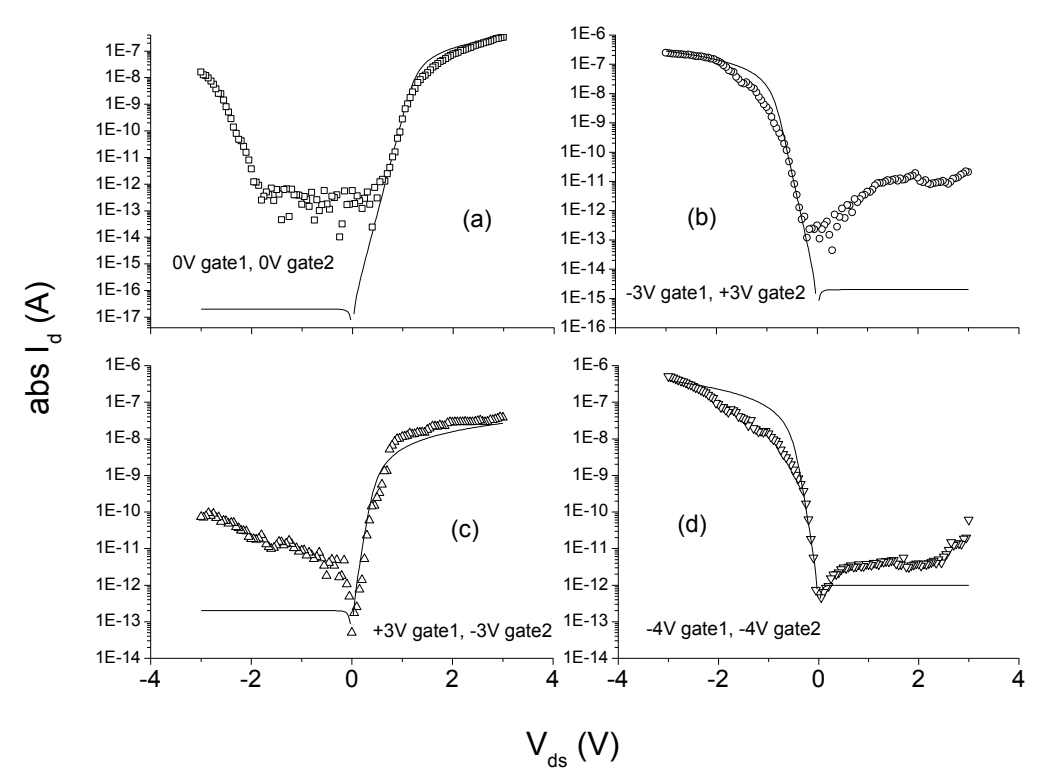

Figure 4. $I_{d^{-}} V_{d s}$ characteristics of the asymmetric contact CNT device at various split gate biases, fitted to the explicit solution of the diode equation. (a) No gate bias applied, the rectification direction is forward, and $\eta=2.2$. (b) Gate $1,2=-3,+3 \mathrm{~V}$, causing inversion of the rectification, $\eta=2.1$. (c) Gate $1,2=+3,-3 \mathrm{~V}$ results in no inversion and $\eta=1.9$. (d) Gate $1,2=-4,-4 \mathrm{~V}$ results in inversion and $\eta$ $=2.4$.

Schottky barrier diodes typically have a larger $I_{0}$ than $\mathrm{p}-\mathrm{n}$ junction diodes as the Schottky barrier height is generally smaller than the barrier height in p-n junction diodes. In Schottky diodes $\eta=1$ indicates there is little or no tunnelling current or depletion layer recombination. Higher $\eta$ values indicate that tunnelling dominates and are associated with higher $I_{0}$ [37]. The saturation current in Schottky diodes is given by [38]

$I_{0}=A^{* *} A T^{2} e^{\left[\frac{-q\left(\Phi_{b}-\Delta \Phi\right)}{k T}\right]}$

where $\Phi_{\mathrm{b}}$ is the Schottky barrier height, $\Delta \Phi$ is the image charge lowering, and $A^{* *}$ is the reduced effective Richardson constant, which is a function of electron rest mass and effective mass and should not be affected by the gate bias. The only factor that should be modified by the gate bias is $\Phi_{\mathrm{b}}$. At zero and equal split gate bias the observed rectification should be caused by the formation of a Schottky diode whilst with opposite split gate biases a p-n junction should be present. In the case of a p-n junction reverse bias current is due to the diffusion current from minority carriers and/or the generation of electron hole pairs in the junction. An ideality factor of 1 represents a saturation current due to diffusion 
current and an ideality factor of 2 represents a saturation current due to generation current, if $\eta>2$, this indicates the junction does not behave as an ideal p-n junction [11, 37, 39]. In symmetrically contacted CNT p-n junctions formed by a split gate Malapanis et al showed that $I_{0}$ decreased exponentially with increasing CNT bandgap [11].

At zero gate bias, shown in Figure 4a, fitting the diode equation indicated an extremely low reverse bias saturation current of $2 \times 10^{-17}$ A. However, in the reverse bias region the detection limit of our measurement system was much larger than this. Additionally, the diode appears to breakdown at high reverse bias. The ideality factor of 2.2 indicates that current transport is dominated by tunnelling. Mobility is an important performance parameter for a FET, and there is a trade-off relationship between mobility and on/off ratio in CNT FETs [40] i.e. high on/off ratios tend to result in low mobility. Using the method detailed in ref [41] we calculate a mobility of $39 \mathrm{~cm}^{2} / \mathrm{Vs}$. Together with the on/off ratio of $1 \times 10^{7}$ our device compares favourably to one of the highest reported mobility and on/off ratio combinations of 35 $\mathrm{cm}^{2} / \mathrm{Vs}$ and $6 \times 10^{6}$ respectively, for CNT thin film transistors from which sequential logic devices were fabricated [41].

The highest achievable open circuit voltage $\left(V_{\mathrm{OC}}\right)$ for any diode is given by [37]

$V_{O C}=\frac{\eta K_{B} T}{q} \ln \left[\frac{I_{S C}}{I_{0}}+1\right]$

Using the values of $I_{0}$ and $\eta$ from the diode equation fitting, $V_{\mathrm{OC}}$ was calculated from $I_{\mathrm{SC}}$ using equation 2 and plotted in Figure $5 \mathrm{~b}$ as $V_{\mathrm{OC}}(\mathrm{calc})$. Inspection indicates that $V_{O C}$ and $I_{S C}$ have the same dependence on incident laser power, both plateauing at $0.28 \mathrm{~V}$ and $41 \mathrm{pA}$ respectively after the laser power density is increased above $250 \mathrm{~kW} / \mathrm{cm}^{2}$, which is probably due to saturation of the diode. $V_{\mathrm{OC}}(\mathrm{calc})$ also follows this trend, plateauing at $0.43 \mathrm{~V}$. The plateauing of $V_{\mathrm{OC}}$ is therefore caused by saturation of the photocurrent which should saturate above a certain exciton generation rate [42]. The measured $V_{\mathrm{OC}}$ should be approximately equal to $V_{b i}$ in this p-n diode. From our diameter estimate (see below) the nanotube bandgap should be $\sim 0.5 \mathrm{eV}$ [43]. This indicates the use of split gates has a relatively large effect on the nanotube band structure. Photocurrent steps indicating charge carriers in the second nanotube subband [44] can be observed in Figure 5a. The average period of the step is $0.43 \mathrm{~V}$, which should be approximately equal to $V_{O C}$ [44], and is in good agreement with $V_{O C}(\mathrm{calc})$. Using $V_{O C}=0.43 \mathrm{~V}$, and the dependence of $V_{O C}$ on CNT diameter suggested by Gabor et al [44], we estimate a CNT diameter of $1.6 \mathrm{~nm}$.

Proc. of SPIE Vol. $898289820 \mathrm{P}-11$ 

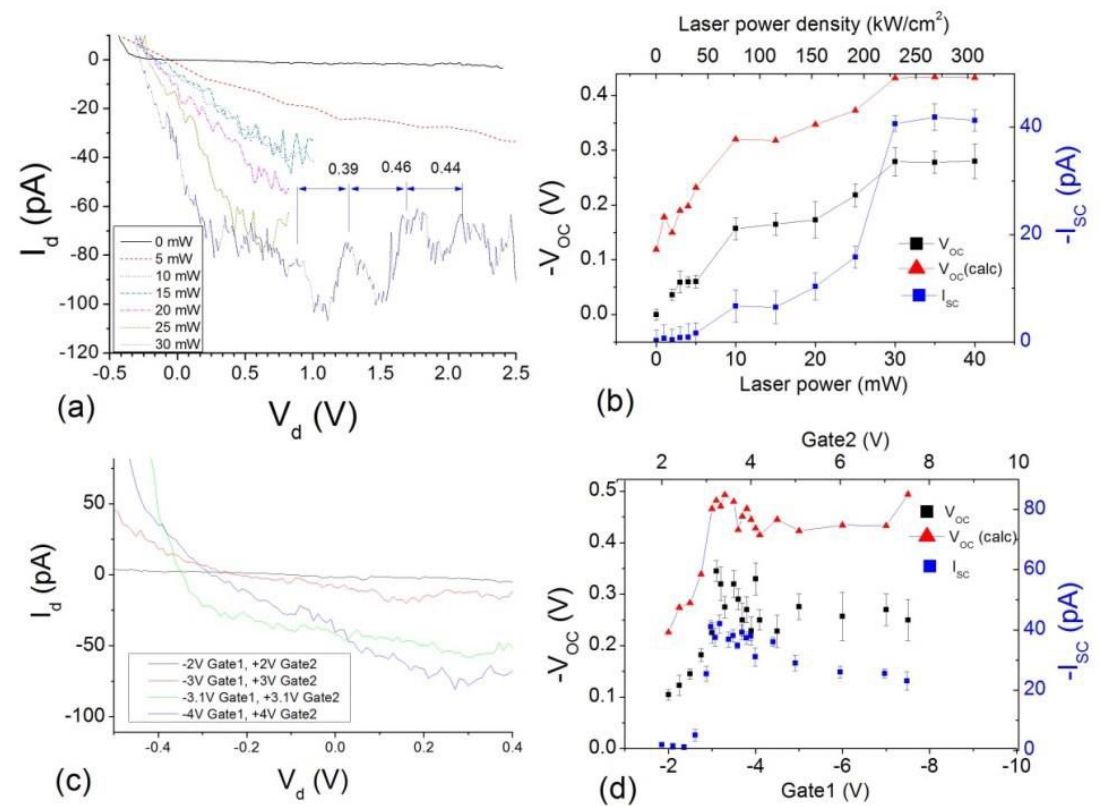

Figure 5. $I_{d}-V_{d s}$ photocurrent characteristics at various laser illumination powers $(\lambda=735 \mathrm{~nm})$ with gate $1,2=-4,+4 V$, the step region for $30 \mathrm{~mW}$ of illumination is marked (a). $V_{\mathrm{OC}}, V_{\mathrm{OC}}(\mathrm{calc})$ and $I_{\mathrm{SC}}$ as a function of laser power with gate $1,2=-4,+4 \mathrm{~V}(\mathrm{~b}) . \mathrm{I}_{\mathrm{d}}-\mathrm{V}_{\mathrm{ds}}$ photocurrent characteristics at various split gate biases with a laser power of $30 \mathrm{~mW}$ (c). $\mathrm{V}_{\mathrm{OC}}$ and $\mathrm{I}_{\mathrm{SC}}$ as a function of split gate bias with a laser power of $30 \mathrm{~mW}(\mathrm{~d})$.

We also measured $I_{d}-V_{d s}$ at various opposite split gate biases with the laser power fixed at $30 \mathrm{~mW}$. Some examples are shown in Figure 5c. Figure $5 \mathrm{~d}$ shows $V_{O C}$ and $I_{S C}$ vs opposite split gate bias where $V_{O C}($ calc) was calculated in the same manner as Figure 5b. $V_{O C}$ increases super-linearly until at around gate $1,2=-3,+3 \mathrm{~V}$ it reaches a maximum of $0.34 \mathrm{~V}$ before plateauing. $I_{O C}$ has a similar dependence to $V_{O C}$ plateauing at gate $1,2=-3,+3 \mathrm{~V}$ and reaching a maximum of 41 pA. However, there is a slight initial decrease as the split gate bias is increased from its minimum. $V_{O C}($ calc) reaches a maximum of $0.49 \mathrm{~V}$, follows the $V_{O C}$ dependence more closely and does not have the initial decrease observed in $I_{S C}$. Examination of the diode fitting parameters used to calculate $V_{O C}\left(\right.$ calc) indicated that this is due to higher $I_{O}$ and $\eta$ at lower split gate bias. Increasing opposite polarity split gate bias should monotonically increase $V_{b i}$ and therefore $V_{O C}$. However, as $V_{O C}$ is dependent on $I_{S C}$, the plateauing of $V_{O C}$ is caused by the saturation of the photocurrent. In a symmetrically contacted split gate CNT diode $I_{S C}$ showed either no dependence or a small increase with increased split gate bias [45]. In an asymmetrically contacted CNT FET with split gates applying opposite polarity bias to the gates 
resulted in photocurrent characteristics similar to those without any gate bias [46]. In a symmetrically contacted single gate CNT FET $I_{S C}$ could be modulated with the gate bias [17]. We calculated the power conversion efficiency $\eta_{\mathrm{c}}$ from $\eta_{\mathrm{c}}=V_{\mathrm{OC}} \times I_{\mathrm{SC}} \times F F \times A_{r} / P_{\text {in }}$, where $F F$ is the fill factor, $A_{r}$ is the ratio of the total illuminated area $\left(1.3 \times 10^{-7} \mathrm{~cm}^{2}\right)$ to the illuminated area of the CNT $\left(1 \mu \mathrm{m} \times 1.6 \mathrm{~nm}=2.6 \times 10^{-11} \mathrm{~cm}^{2}\right) . \eta_{c}$ had a maximum of $\sim 1 \times 10^{-6}$ which compares to $2 \times 10^{-3}$ for a suspended single nanotube split gate diode using a $1.5 \mu \mathrm{m}$ laser [45]. The difference may be due to our CNTs being unsuspended and grown by CVD which means they are in intimate contact with the substrate which can leave them susceptible to defects. The bandwidth of a photodiode depends, broadly, on its depletion width which increases with $V_{b i}$ [37]. We would therefore expect the bandwidth of our photodiode to be higher for low split gate bias which has lower $V_{b i}$. With increased split gate bias the power conversion efficiency increases. The split gate bias may therefore be used to switch between high bandwidth/low efficiency and low bandwidth/high efficiency operation, overcoming the bandwidthefficiency trade-off of our photodiode.

\section{Conclusions}

The $I_{d^{-}} V_{d s}$, EL and photocurrent characteristics of CNT FETs with split gate geometry can be enhanced and tuned by applying specific split gate biases. The symmetric contact device has symmetric $I_{d}-V_{d s}$ characteristics; $I_{\mathrm{d}}$ changes by $5 \%$ in the expected direction when operated in forward or reverse bias configurations. Ambipolar transfer characteristics were observed with an on/off ratio of $\sim 2$. The modulation depth of $I_{\mathrm{d}}$ is considerably higher when the split gates have equal rather than opposite bias. We suggest that this is due to trapping of carriers by band bending. Six EL bands could be observed with the strongest band peaking at $0.86 \mathrm{eV}$ with a FWHM of $87 \mathrm{meV}$ at $V_{d s}=-4 \mathrm{~V}$ and gate $1,2=-15,+15$ $\mathrm{V}$, indicating that hot carriers are not involved in the EL process. We estimate the power and quantum efficiency of the EL to be around $1 \times 10^{-6}$ and $1 \times 10^{-5}$ respectively. With a fixed $V_{d s}$, increasing the opposite split gate bias caused the EL to increase monotonically from zero, indicating an ambipolar emission mechanism. With a fixed opposite split gate bias the dependence of EL on $V_{d s}, I_{d} V_{d s}$ or $I_{d}$ displays a threshold, indicating a unipolar emission mechanism. We propose that this is due to a larger Schottky barrier height for holes.

When operated as an FET the single CNT asymmetric contact device has an on/off ratio of $1 \times 10^{7}$ and a mobility of 39 $\mathrm{cm}^{2} / \mathrm{Vs}$. When operated as a diode, without any gate bias, it has an $I_{0}$ and $\eta$ of $2 \times 10^{-17} \mathrm{~A}$ and 2.2 , respectively. Applying negative bias to both gates, or opposite polarity biases to gates 1 and 2, respectively, causes inversion of the diode. When 
operated as a photodiode we estimate a power conversion efficiency of $1 \times 10^{-6}$. With gate $1,2=-4,+4 \mathrm{~V} I_{S C}$ and $V_{O C}$ plateau at $41 \mathrm{pA}$ and $0.28 \mathrm{~V}$ respectively when the laser power density is increased above $240 \mathrm{~kW} / \mathrm{cm}^{2}$. This is caused by saturation of the diode. Steps with a period of $0.43 \mathrm{~V}$ can be observed in $I_{\mathrm{d}}-V_{\mathrm{ds}}$ when the laser power density is 240 $\mathrm{kW} / \mathrm{cm}^{2}$. The period of these steps should be approximately equal to $V_{O C}$. When $V_{O C}$ is calculated from $I_{S C}$ and diode fitting parameters, $0.43 \mathrm{~V}$ is also obtained. Using $V_{O C}=0.43 \mathrm{~V}$ we estimate a CNT diameter of $1.6 \mathrm{~nm}$. With a laser power density of $240 \mathrm{~kW} / \mathrm{cm}^{2}$ increasing opposite polarity split gate bias causes $I_{S C}$ and $V_{O C}$ to increase, then plateau at $41 \mathrm{pA}$ and $0.34 \mathrm{~V}$ respectively at gate $1,2=-3,+3 \mathrm{~V}$. The ability to control $V_{O C}$ could be used to switch between high bandwidth/low efficiency and low bandwidth/high efficiency operation.

\section{References}

1. A. Javey, J. Guo, Q. Wang, M. Lundstrom, and H. J. Dai, "Ballistic carbon nanotube field-effect transistors," Nature 424, 654-657 (2003).

2. Z. Yao, C. L. Kane, and C. Dekker, "High-Field Electrical Transport in Single-Wall Carbon Nanotubes," Phys. Rev. Lett. 84, 2941-2944 (2000).

3. T. Mueller, M. Kinoshita, M. Steiner, V. Perebeinos, A. A. Bol, D. B. Farmer, and P. Avouris, "Efficient narrow-band light emission from a single carbon nanotube p-n diode," Nat. Nanotechnol. 5, 27-31 (2010).

4. A. Högele, C. Galland, M. Winger, and A. Imamoğlu, "Photon Antibunching in the Photoluminescence Spectra of a Single Carbon Nanotube," Phys. Rev. Lett. 100, 217401 (2008).

5. J. Lee, H. Kim, S. J. Kahng, G. Kim, Y. W. Son, J. Ihm, H. Kato, Z. W. Wang, T. Okazaki, H. Shinohara, and Y. Kuk, "Bandgap modulation of carbon nanotubes by encapsulated metallofullerenes," Nature 415, 1005-1008 (2002).

6. J. Zaumseil, X. N. Ho, J. R. Guest, G. P. Wiederrecht, and J. A. Rogers, "Electroluminescence from Electrolyte-Gated Carbon Nanotube Field-Effect Transistors," ACS Nano 3, 2225-2234 (2009).

7. M. Freitag, J. Chen, J. Tersoff, J. C. Tsang, Q. Fu, J. Liu, and P. Avouris, "Mobile ambipolar domain in carbon-nanotube infrared emitters," Phys. Rev. Lett. 93, 076803 (2004).

8. J. U. Lee, P. P. Gipp, and C. M. Heller, "Carbon nanotube p-n junction diodes," Appl. Phys. Lett. 85, 145-147 (2004).

9. K. Bosnick, N. Gabor, and P. McEuen, "Transport in carbon nanotube p-i-n diodes," Appl. Phys. Lett. 89, 163121 (2006).

10. C. H. Liu, C. C. Wu, and Z. H. Zhong, "A Fully Tunable Single-Walled Carbon Nanotube Diode," Nano Lett. 11, $1782-1785$ (2011).

11. A. Malapanis, D. A. Jones, E. Comfort, and J. U. Lee, "Measuring Carbon Nanotube Band Gaps through Leakage Current and Excitonic Transitions of Nanotube Diodes," Nano Lett. 11, 1946-1951 (2011).

12. S. M. Bachilo, M. S. Strano, C. Kittrell, R. H. Hauge, R. E. Smalley, and R. B. Weisman, "Structure-assigned optical spectra of single-walled carbon nanotubes," Science 298, 2361-2366 (2002).

13. F. Leonard, Physics of Carbon Nanotube Devices (Elsevier Science, New York, 2008).

14. Y. Jung, X. Li, N. K. Rajan, A. D. Taylor, and M. A. Reed, "Record High Efficiency Single-Walled Carbon Nanotube/Silicon p-n Junction Solar Cells," Nano Lett. 13, 95-99 (2012).

15. Y. Jia, A. Cao, X. Bai, Z. Li, L. Zhang, N. Guo, J. Wei, K. Wang, H. Zhu, D. Wu, and P. M. Ajayan, "Achieving High Efficiency Silicon-Carbon Nanotube Heterojunction Solar Cells by Acid Doping," Nano Lett. 11, 1901-1905 (2011).

16. S. Konabe and S. Okada, "Enhanced photocurrent in single-walled carbon nanotubes by exciton interactions," Appl. Phys. Lett. 102, 113110 (2013).

17. Y. H. Ahn, A. W. Tsen, B. Kim, Y. W. Park, and J. Park, "Photocurrent imaging of p-n junctions in ambipolar carbon nanotube transistors," Nano Lett. 7, 3320-3323 (2007).

18. N. Fukuoka, Y. Mizutani, S. Naritsuka, T. Maruyama, and S. Iijima, "Low-Temperature Synthesis of Single-Walled Carbon Nanotubes in a High Vacuum Using Pt Catalyst in Alcohol Gas Source Method," Jpn. J. Appl. Phys. 51, 06FD23 (2012).

19. T. Mizutani, Y. Nosho, and Y. Ohno, "Electrical properties of carbon nanotube FETs," J. Phys. Conf. Ser. 109, 012002 (2008).

20. Y. Mizutani, N. Fukuoka, S. Naritsuka, T. Maruyama, and S. Iijima, "Single-walled carbon nanotube synthesis on SiO2/Si substrates at very low pressures by the alcohol gas source method using a Pt catalyst," Diam. Relat. Mat. 26, 78-82 (2012).

21. S. Noda, H. Sugime, T. Osawa, Y. Tsuji, S. Chiashi, Y. Murakami, and S. Maruyama, "A simple combinatorial method to discover Co-Mo binary catalysts that grow vertically aligned single-walled carbon nanotubes," Carbon 44, 1414-1419 (2006). 
22. T. Brintlinger, Y.-F. Chen, T. Durkop, E. Cobas, M. S. Fuhrer, J. D. Barry, and J. Melngailis, "Rapid imaging of nanotubes on insulating substrates," Appl. Phys. Lett. 81, 2454-2456 (2002).

23. W. Kim, A. Javey, O. Vermesh, O. Wang, Y. M. Li, and H. J. Dai, "Hysteresis caused by water molecules in carbon nanotube field-effect transistors," Nano Lett. 3, 193-198 (2003).

24. M. S. Fuhrer, B. M. Kim, T. Dürkop, and T. Brintlinger, "High-Mobility Nanotube Transistor Memory," Nano Lett. 2, 755-759 (2002).

25. M. Kinoshita, M. Steiner, M. Engel, J. P. Small, A. A. Green, M. C. Hersam, R. Krupke, E. E. Mendez, and P. Avouris, "The polarized carbon nanotube thin film LED," Opt. Express 18, 25738-25745 (2010).

26. V. Perebeinos, J. Tersoff, and P. Avouris, "Scaling of Excitons in Carbon Nanotubes," Phys. Rev. Lett. 92, 257402 (2004).

27. R. B. Weisman and S. M. Bachilo, "Dependence of optical transition energies on structure for single-walled carbon nanotubes in aqueous suspension: An empirical Kataura plot," Nano Lett. 3, 1235-1238 (2003).

28. N. Hibino, S. Suzuki, H. Wakahara, Y. Kobayashi, T. Sato, and H. Maki, "Short-Wavelength Electroluminescence from SingleWalled Carbon Nanotubes with High Bias Voltage," ACS Nano 5, 1215-1222 (2011).

29. L. Xie, H. Farhat, H. Son, J. Zhang, M. S. Dresselhaus, J. Kong, and Z. Liu, "Electroluminescence from Suspended and OnSubstrate Metallic Single-Walled Carbon Nanotubes," Nano Lett. 9, 1747-1751 (2009).

30. D. Mann, Y. K. Kato, A. Kinkhabwala, E. Pop, J. Cao, X. R. Wang, L. Zhang, Q. Wang, J. Guo, and H. J. Dai, "Electrically driven thermal light emission from individual single-walled carbon nanotubes," Nat. Nanotechnol. 2, 33-38 (2007).

31. R. B. Capaz, C. D. Spataru, P. Tangney, M. L. Cohen, and S. G. Louie, "Temperature Dependence of the Band Gap of Semiconducting Carbon Nanotubes," Phys. Rev. Lett. 94, 036801 (2005).

32. E. Adam, C. M. Aguirre, L. Marty, B. C. St-Antoine, F. Meunier, P. Desjardins, D. Menard, and R. Martel, "Electroluminescence from single-wall carbon nanotube network transistors," Nano Lett. 8, 2351-2355 (2008).

33. M. Freitag, V. Perebeinos, J. Chen, A. Stein, J. C. Tsang, J. A. Misewich, R. Martel, and P. Avouris, "Hot carrier electroluminescence from a single carbon nanotube," Nano Lett. 4, 1063-1066 (2004).

34. P. Avouris, M. Freitag, and V. Perebeinos, "Carbon-nanotube photonics and optoelectronics," Nat. Photonics 2, 341-350 (2008).

35. M. Freitag, J. C. Tsang, J. Kirtley, A. Carlsen, J. Chen, A. Troeman, H. Hilgenkamp, and P. Avouris, "Electrically excited, localized infrared emission from single carbon nanotubes," Nano Lett. 6, 1425-1433 (2006).

36. M. A. Hughes, K. P. Homewood, R. J. Curry, Y. Ohno, and T. Mizutani, "An ultra-low leakage current single carbon nanotube diode with split-gate and asymmetric contact geometry," Appl. Phys. Lett. 103, 133508 (2013).

37. S. M. Sze and K. K. Ng, Physics of Semiconductor Devices (Wiley, 2006).

38. D. Perello, B. Dong Jae, M. J. Kim, C. Dongkyu, J. Seung Yol, K. Bo Ram, Y. Woo Jong, L. He Younge, and Y. Minhee, "Quantitative Experimental Analysis of Schottky Barriers and Poole-Frenkel Emission in Carbon Nanotube Devices," Nanotechnology, IEEE Transactions on 8, 355-360 (2009).

39. J. U. Lee, "Band-gap renormalization in carbon nanotubes: Origin of the ideal diode behavior in carbon nanotube p-n structures," Phys. Rev. B 75, 075409 (2007).

40. R. Nouchi, H. Tomita, A. Ogura, H. Kataura, and M. Shiraishi, "Logic circuits using solution-processed single-walled carbon nanotube transistors," Appl. Phys. Lett. 92, 253507 (2008).

41. D. M. Sun, M. Y. Timmermans, Y. Tian, A. G. Nasibulin, E. I. Kauppinen, S. Kishimoto, T. Mizutani, and Y. Ohno, "Flexible high-performance carbon nanotube integrated circuits," Nat. Nanotechnol. 6, 156-161 (2011).

42. S. N. Mohammad and S. T. H. Abidi, "Theory of saturation photocurrent and photovoltage in p-n junction solar cells," J. Appl. Phys. 61, 4909-4919 (1987).

43. H. Kataura, Y. Kumazawa, Y. Maniwa, I. Umezu, S. Suzuki, Y. Ohtsuka, and Y. Achiba, "Optical properties of single-wall carbon nanotubes," Synthetic Metals 103, 2555-2558 (1999).

44. N. M. Gabor, Z. H. Zhong, K. Bosnick, J. Park, and P. L. McEuen, "Extremely Efficient Multiple Electron-Hole Pair Generation in Carbon Nanotube Photodiodes," Science 325, 1367-1371 (2009).

45. J. U. Lee, "Photovoltaic effect in ideal carbon nanotube diodes," Appl. Phys. Lett. 87, 073101 (2005).

46. H. Z. Chen, N. Xi, K. W. C. Lai, L. L. Chen, R. G. Yang, and B. Song, "Gate dependent photo-responses of carbon nanotube field effect phototransistors," Nanotechnology 23, 385203 (2012). 